\title{
Quasi-autonomous thermal model reduction for steady-state problemsin space systems
}

\section{Germán Fernández-Rico, Isabel Pérez-Grande, Angel Sanz-Andres, Ignacio Torralbo , Joachim Woch}

\begin{abstract}
A matrix method is developed to reduce the number of elements of spacecraft thermal mathematical models based on the lumped parameter method. The aim of this method is to achieve a satisfactory thermal model reduction for steady-state problems, in an automatic way, while preserving the physical meaning of the system and the main characteristics of the model. The simplicity of the method, and the computational cost, are also taken into account. The reduction process is based on the manipulation of the conductive coupling matrix, which is treated as a sparse graph adjacency matrix. Then, a depth-first search algorithm is used to find the strongly connected components, which define the condensed nodes. Finally, all the thermal entities are reduced, and the results from the condensed model are compared to those from the detailed one. The entire reduction process is tested on a real thermal model, showing a good performance. In the conclusions section the characteristics and limitations of this method are shown.
\end{abstract}

\section{Introduction and state of the art}

Spacecraft thermal control addresses the problem of keeping space vehicles and their components temperatures within the appropriate range [1-3]. Tests and analyses are used together for the study and the design of a thermal control subsystem. In the earliest stages of the design, the preliminary studies can be performed using analytical calculations [4-9]. But as the system becomes more complex and detailed, it is necessary to rely on other tools. While the tests allow the design to be verified, they are very complex and expensive. Therefore, in recent years, thermal simulation has become more and more relevant. In Europe, the standard tool for thermal analysis in the space industry is ESATAN-TMS [10].
When working with thermal models, it is very usual to have very detailed models (for typical lumped parameter solvers, it is in the range of $10^{3}-10^{4}$ nodes) for the element/unit which is being studied. Then, this element might have to be included in a higher hierarchy model, or it could be necessary to carry out extensive simulations (e.g. transient and sensitivity analysis). To keep the size of the model under reasonable limits ( $<10^{5}$ nodes) and save analysis time, in all the aforementioned cases it is highly recommendable to reduce the number of nodes of the thermal model under study. The reduced thermal model must behave as the detailed one (in terms of temperatures and heat fluxes through the boundaries), while having a smaller number of nodes, so-called "size".

The classical approach to deal with this situation is to set up a reduced geometrical mathematical model (RGMM). The reduced thermal mathematical model (RTMM) is built on a userdefinition basis. This means the modeler builds the RGMM, and 


\section{Nomenclature}

A adjacency matrix, $n_{d} \times n_{d}$

B change of basis matrix, $n_{d} \times n_{d}$

C thermal capacity vector, $n \times 1(\mathrm{~J} / \mathrm{K})$

$c_{p} \quad$ specific heat capacity $(\mathrm{J} /(\mathrm{kg} \mathrm{K}))$

D distance matrix, $n_{d} \times n_{d}(\mathrm{~m})$

G thermal capacity matrix, $n_{d} \times n_{d}(\mathrm{~J} / \mathrm{K})$

H heat flux vector, $n_{b} \times 1(\mathrm{~W})$

I identity matrix, $n \times n$

K conductive coupling matrix, $n \times n(\mathrm{~W} / \mathrm{K})$

$L \quad$ distance between node center and interface $(\mathrm{m})$

$n \quad$ number of nodes

P restriction matrix, $n_{d} \times n_{c}$

$p_{f} \quad$ dimensionless filtering threshold

q heat flux difference vector between detailed and reduced model results, $n_{b} \times 1$ (\% or $\mathrm{W}$ )

$q_{\max } \quad$ maximum allowable heat flux difference (\% or $\mathrm{W}$ )

$q_{\text {lim }} \quad$ heat flux limit for separation between relative and absolute values of heat flux difference (W)

Q thermal loads vector, $n \times 1(\mathrm{~W})$

$r_{r} \quad$ reduction ratio

$\mathbf{R} \quad$ radiative coupling matrix, $n \times n\left(\mathrm{~m}^{2}\right)$

$S \quad$ node cross-section $\left(\mathrm{m}^{2}\right)$

$\mathbf{S}_{\mathbf{c n}} \quad$ connected components label array, $n_{d} \times 1$

$t \quad$ thickness $(\mathrm{m})$

T temperature vector, $n \times 1(\mathrm{~K})$

$v \quad$ node volume $\left(\mathrm{m}^{3}\right)$

$w \quad$ node width $(\mathrm{m})$

X node spatial coordinates $(x, y, z), n_{d} \times 3(\mathrm{~m})$

Greek symbols

$\delta \quad$ vector of temperature differences between derived and calculated values $(\mathrm{K}), n_{c} \times 1$

$\delta_{\max } \quad$ maximum allowable temperature difference (K)

$\Delta T_{\max } \quad$ temperature difference threshold (K)

$\lambda$ thermal conductivity $(\mathrm{W} /(\mathrm{m} \mathrm{K}))$

$\mu \quad$ parameter $\left(\mathrm{m}^{2} / \mathrm{s}\right)$

$\rho \quad$ density $\left(\mathrm{kg} / \mathrm{m}^{3}\right)$

$\sigma \quad$ Stefan-Boltzmann constant $\left(\mathrm{W} /\left(\mathrm{m}^{2} \mathrm{~K}^{4}\right)\right)$ temperature difference matrix, $n \times n(\mathrm{~K})$

Accents

- $\quad$ derived values

$\wedge \quad$ calculated values

$\sim \quad$ dimensionless values

\section{Subscripts}

b boundary node

c condensed model

$\mathrm{cb} \quad$ conductive to boundary nodes

d detailed model

$\mathrm{rb} \quad$ radiative to boundary nodes

sort sorted

\section{Superscripts}

B including marked boundary nodes

D detailed model

F filtered

I including marked internal boundary nodes

$\mathrm{R} \quad$ reduced model

$\mathrm{S} \quad$ sizing

T transposed

* linearized

\section{Abbreviations}

CSR Compressed Storage by Rows

GMM Geometrical Mathematical Model

ESA European Space Agency

FPA Focal Plane Assembly

MLI Multi-Layer Insulation

PHI Polarimetric Helioseismic Imager

RGMM Reduced Geometrical Mathematical Model

RTMM Reduced Thermal Mathematical Model

SCC Strongly Connected Components

TMD Thermal Model Data

TMM Thermal Mathematical Model according to its definition, the nodes from the detailed thermal mathematical model (DTMM) are condensed into RTMM nodes. Then, the general practice is to get two snapshots of the DTMM (usually hot and cold operational worst cases) and, by tuning the conductive couplings and the heat loads, try to match the results from the RTMM and the DTMM. This process is time consuming, and tends to be error-prone.

Some authors and companies have developed a number of algorithms and computer codes to automatize this reduction process. For instance, Bernard et al. [11-13] have developed the TMRT (thermal model reduction tool) software. This software is intended to reduce large mathematical models. The program is based on a Guyan-Irons reduction, and it seems to work well, but it needs the reduction to be pre-defined by the engineer. This approach requires essentially defining the reduction scheme a priori. A very similar approach can be found in [14-16]. Deiml et al. [17] have carried out a wide analysis of the different techniques that could be applied to spacecraft thermal model reduction. They focus on nonlinear methods, which can achieve reductions with very good correlation, at the expense of losing part of the physical interpretation of the problem. They are also the only ones that also deal with the reduction of the geometrical mathematical model (GMM).

The main goals and points of the quasi-autonomous thermal reduction process presented here are: a. Reduce the thermal model taking into account the different quasi-isothermal zones. By grouping similar-temperature nodes, it is possible to reduce the error produced by reducing the matrices. It can be supposed that parts which are strongly connected (larger conductive couplings) will be more isothermal than other parts which are weakly connected.

b. The model reduction should be done in a quasi-automatic way. This means, the only parameters that need to be defined are the maximum allowable temperature and heat fluxes differences, as well as the desired reduction ratio.

c. The reduction process will depend on the temperatures and boundary conditions. Having more scenarios (e.g. hot and cold operational cases) will constrain the problem more.

d. In engineering applications, it is essential to preserve the physical interpretation of the model elements, as well as the physical characteristics, to be able to take design decisions changing physically achievable parameters. This implies having positive thermal couplings, to preserve the boundary nodes, the heat loads, the symmetry in the coupling matrices, and the conductive physical paths.

The work presented hereafter is partially inspired by the large resistor networks reduction methods $[18,19]$. 


\section{Thermal model}

The lumped parameter method thermal mathematical model is described by Eq. (1), one energy balance equation per node

$C_{i} \frac{\mathrm{d} T_{i}}{\mathrm{~d} t}=\sum_{j=1}^{n_{d}}\left[K_{i j}\left(T_{j}-T_{i}\right)+R_{i j}\left(T_{j}^{4}-T_{i}^{4}\right)\right]+Q_{i}, \quad i=1, \ldots, n_{d}, \quad i \neq j$,

where $C_{i}$ is the thermal capacitance of node $i, K_{i j}$ and $R_{i j}$ the conductive and radiative couplings between nodes $i$ and $j$, respectively, and $Q_{i}$ the heat load on node $i$ ( $R_{i j}$ include the Stefan-Boltzmann constant, $\sigma$ ). The sign criteria is $Q_{i}>0$ when heat is entering the node. The coefficients of these equations are usually generated by means of a thermal analysis software package. For the development and execution of the reduction algorithm, regardless of the thermal modelling software used, all the variables which appear in (1) are considered as available inputs. These are $\mathbf{K}$ (conductive coupling matrix), $\mathbf{R}$ (radiative coupling matrix), $\mathbf{T}$ (temperature vector), $\mathbf{C}$ (node thermal capacity vector) and $\mathbf{Q}$ (node heat load vector). Also the node spatial coordinate matrix, $\mathbf{X}$, is considered to be known. Although the materials of the spacecraft system are obviously known, since the aim of this work is to reduce a detailed thermal model already set up, some information is considered not to be available (e.g. specific heat capacities, thermal conductivities, etc.).

\section{Reduction process}

\subsection{Scope}

Reducing linear models (those exclusively made of conductive couplings) is quite straightforward [18], whereas reducing nonlinear models (those which include radiative terms) can lead to excessive temperature deviations between the detailed and the reduced models, if the radiative terms dominate the problem. It is considered here that two nodes can be condensed only if they are connected by material medium, so that the physical meaning is preserved. That is why only the conductive couplings matrix $\mathbf{K}$ is taken into account to run the reduction algorithm. Once the model has been reduced, the radiative couplings matrix $\mathbf{R}$ is reduced using the same scheme and the problem can be linearized and solved.

A flow diagram of the reduction process that is explained in the subsequent sections is shown in Fig. 1. The goal of the reduction process is to find, in an autonomous way, the scheme that groups the thermal nodes into condensed thermal nodes. In particular, the aim is to transform the matrix system dimension from $n_{d}$ (number of nodes of the detailed model) to $n_{c}$ (number of nodes of the reduced model). The relation between $n_{d}, n_{c}$ and $n_{b}$ (number of boundary nodes, invariant in the reduction process) is here called reduction ratio $r_{r}$, which is a measure of the reduction degree. $r_{r}$ is defined as

$r_{r}=1-\frac{n_{c}-n_{b}}{n_{d}-n_{b}}$

as boundary nodes are not considered in the reduction process. $r_{r}$ ranges from 0 to $1 ; 0$ indicates that the reduced and the detailed models are the same, whereas 1 represents complete reduction, as only the boundary nodes remain in the reduced model.

To transform the system, a matrix $\mathbf{A}$ is built which indicates the connection between nodes, taking into account the conductive coupling $\mathbf{K}$ and the temperature difference between nodes $\boldsymbol{\Theta}$. Matrix $\mathbf{A}$ is treated as an adjacency matrix, on which the strongly connected components (SCC) algorithm [20-22] is used. The resulting reduction scheme (represented by restriction matrix $\mathbf{P}$ ) is applied to the thermal problem, reducing the dimension of matrices $\mathbf{K}^{\mathbf{R}}, \mathbf{R}^{\mathbf{R}}, \mathbf{Q}^{\mathbf{R}}$. Then this system is solved, and the calculated temperatures $\widehat{\mathbf{T}}^{\mathbf{R}}$ and heat fluxes $\widehat{\mathbf{H}}^{\mathbf{R}}$ are compared to those derived from the detailed thermal model, $\overline{\mathbf{T}}^{\mathbf{D}}$ and $\mathbf{H}^{\mathbf{D}}$. An iteration loop is in charge of achieving the requirements imposed on temperature and heat flux differences. All the steps described above have been programmed with Python 3.4 and the Python-based scientific ecosystem SciPy. Compressed Storage by Rows (CSR) sparse matrices have been used in order to save memory and make the code more efficient.

\subsection{Processing of conductive coupling matrix $\boldsymbol{K}$}

The conductive couplings between the different nodes in a thermal model are calculated based on geometrical and physical properties, as an application of Fourier's law in a lumped parameter model [23-25]. Usually, the values generated in this way are within quite a wide range (approx. $10^{-5}$ to $10^{+3} \mathrm{~W} / \mathrm{K}$ ). One of the most important contributions of this study is to realize that to compare and classify the different values as "strong" or "weak" conductors, one has to take into account not only the values themselves, but also the geometrical and physical properties which have produced such values. It should be considered that, when constructing a thermal model, different spatial scales are frequently used for different parts of the geometrical model, and they largely influence the values of the couplings. For instance, anticipating that metallic parts, such as structural panels, will be quasiisothermal, one can produce a coarser mesh. On the other hand, modelling printed circuit boards, where it could be important to distribute accurately the electric power sources, can lead to a finer mesh. This situation can produce thermal models with a great disparity of node sizes, and therefore of thermal coupling values. The diversity of bulk materials in the model construction, the simulation of thermal contacts at the bolted or glued interfaces, and other effects, greatly influence the inhomogeneity of the conductors' values. If one wants to group the nodes based on how "strong" the conductors are, it is necessary to assess the relationship between the conductor's actual value and how big this conductor could be, based on the available magnitudes. To help making this comparison, a dimensionless conductive coupling matrix, $\widetilde{\mathbf{K}}$, is built.

\subsubsection{Building the dimensionless coupling matrix $\widetilde{\mathbf{K}}$}

To obtain the dimensionless conductive coupling matrix $\widetilde{\mathbf{K}}$ typical values of $K_{i j}$ are needed to be used as a reference. These reference values are grouped inside the conductive coupling sizing matrix, $\mathbf{K}^{\mathbf{S}}$. First, let us build the conductive coupling sizing matrix, $\mathbf{K}^{\mathbf{S}}$. As we want to identify which nodes should be condensed, we are interested in having orders of magnitude for the conductive couplings in $\mathbf{K}^{\mathbf{S}}$ based on the size of the contact and the materials involved. This matrix defines a potential value for a typical coupling for each conductive coupling existing in the model. For each non-zero $i j$ element of matrix $\mathbf{K}$, the conductive coupling between node $i$ and $j$ is defined as follows

$K_{i j}^{s}=\frac{1}{\frac{L_{i}}{\lambda_{i} S_{i}}+\frac{L_{j}}{\lambda_{j} S_{j}}}$,

where $\lambda_{i}$ and $\lambda_{j}$ are the thermal conductivities, $L_{i}$ and $L_{j}$ the distances between the node centers and the interface, and $S_{i}$ and $S_{j}$ the cross section for the conductive coupling calculation. All the geometric magnitudes involved are shown in Fig. 2.

The thermal conductivities $\lambda_{i}$ are not explicitly included in the inputs mentioned in Section 2, so they are unknown. To estimate the sizing matrix $\mathbf{K}^{\mathbf{S}}$, a reference value, say $\lambda$, is used for all the conductors. This generic value itself does not matter and does not 


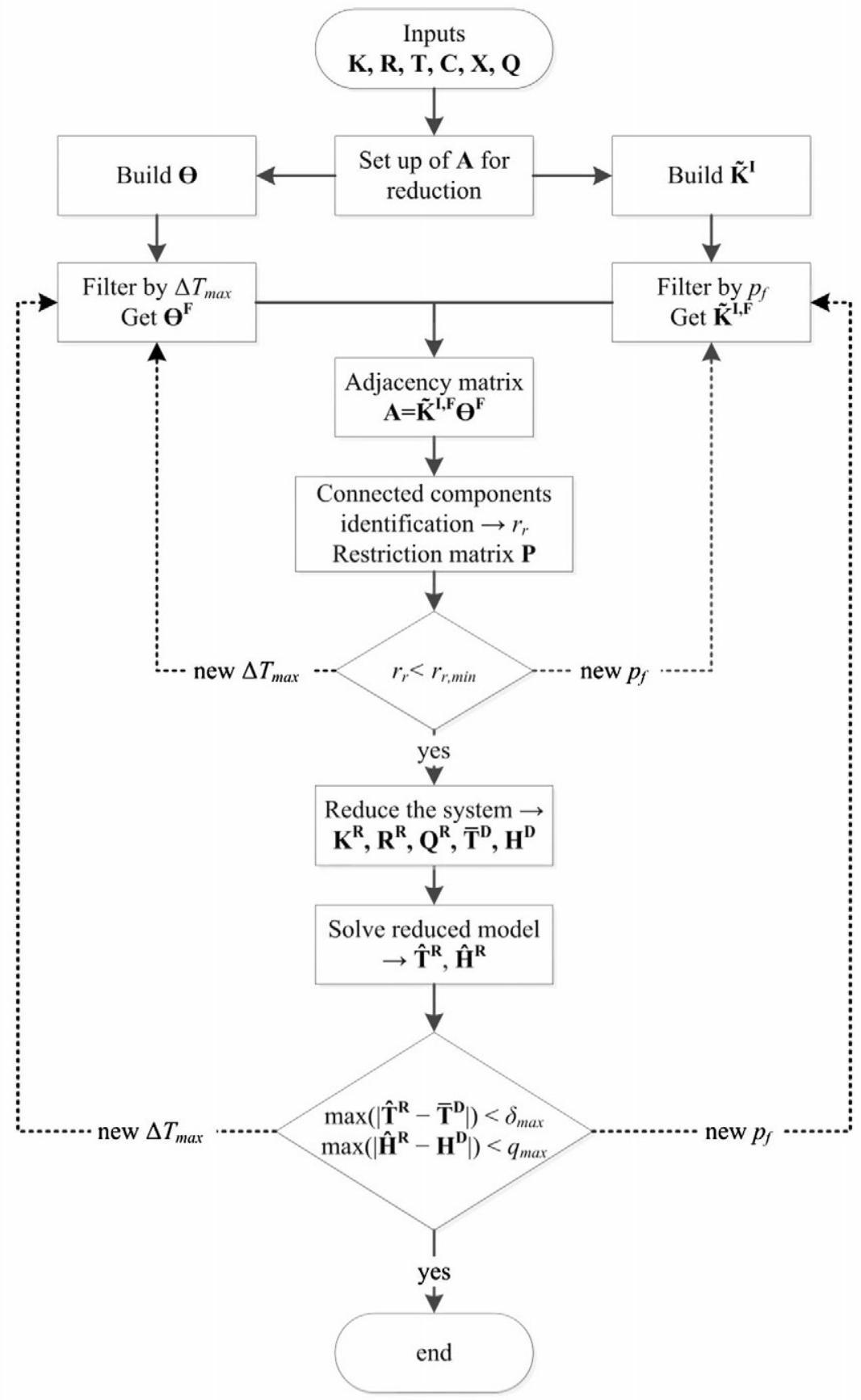

Fig. 1. Reduction process flow diagram.

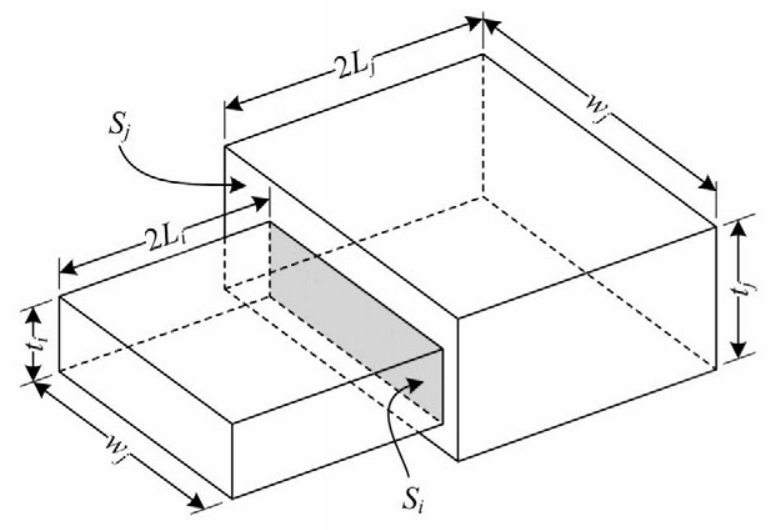

Fig. 2. Conductive coupling calculation scheme. influence the results. It is intended just to obtain values with thermal engineering meaning. Therefore

$K_{i j}^{S}=\frac{\lambda}{\frac{L_{i}}{S_{i}}+\frac{L_{i}}{S_{j}}}$.

$S_{i}, S_{j}$ are also unknown, and estimated as follows. Let us consider the thermal capacities for the nodes $i$ and $j$

$$
\left\{\begin{array}{l}
C_{i}=\rho_{i} c_{p_{i}} v_{i}=\rho_{i} c_{p_{i}} w_{i} t_{i} 2 L_{i} \\
C_{j}=\rho_{j} c_{p_{j}} v_{j}=\rho_{j} c_{p_{j}} w_{j} t_{j} 2 L_{j}
\end{array},\right.
$$

where, as for the thermal conductivities, $\rho$ and $c_{p}$ cannot be inferred from the input data. Again, standard values are used for these magnitudes. $S_{i}$ and $S_{j}$ can be defined as

$S_{i}=w_{i} t_{i} ; \quad S_{j}=w_{j} t_{j}$. 
Although $w$ and $t$ are unknown magnitudes, they can be obtained from (5). Isolating the group $w \cdot t$, one gets

$w_{j} t_{i}=\frac{C_{i}}{2 \rho c_{p} L_{i}} ; \quad w_{j} t_{j}=\frac{C_{j}}{2 \rho c_{p} L_{j}}$.

Substituting (6) and (7) into (4)

$K_{i j}^{\varsigma}=\frac{\lambda}{\frac{L_{i}}{w_{i} t_{j}}+\frac{L_{j}}{w_{j} t_{j}}}=\frac{\lambda}{2 \rho c_{p}} \cdot \frac{1}{\frac{L_{i}^{2}}{C_{i}}+\frac{L_{j}^{2}}{C_{j}}}$.

We can try to estimate the values for $L_{i}$ and $L_{j}$. From $\mathbf{X}$ the distance between nodal centers $i$ and $j, D_{i j}$, can be calculated. It is assumed that the nodal geometries are similar, and therefore the distances $L_{i}$ and $L_{j}$ are of the same order of magnitude. Then

$L_{i} \sim L_{j} \rightarrow L_{i}=L_{j}=\frac{D_{i j}}{2}$.

And consequently, using (8) and (9) we get

$K_{i j}^{s}=\frac{2 \lambda}{\rho c_{p}} \cdot \frac{1}{D_{i j}^{2}} \cdot \frac{1}{\frac{1}{C_{i}}+\frac{1}{C_{j}}}=\mu \cdot \frac{G_{i j}}{D_{i j}^{2}}$,

where the parameter $\mu$ is defined as

$\mu=\frac{2 \lambda}{\rho c_{p}}$,

and

$G_{i j}=\frac{1}{\frac{1}{C_{i}}+\frac{1}{C_{j}}}$.

The sizing matrix $\mathbf{K}^{\mathbf{S}}$ is obtained from (8) by calculating the thermal capacity and distance matrices, $\mathbf{G}$ and $\mathbf{D}$, which are symmetric, together with the parameter $\mu$. The choice of the parameter $\mu$ does not affect the construction of the dimensionless matrix $\widetilde{\mathbf{K}}$, as the same value is used for all the elements. But it is important to choose a realistic value, so that the sizing conductive coupling matrix $\mathbf{K}^{\mathbf{S}}$ has realistic values as well. Assuming typical values, such as $\lambda=50 \mathrm{~W} /(\mathrm{m} \mathrm{K}), \rho=3000 \mathrm{~kg} / \mathrm{m}^{3}$ and $c_{p}=1000 \mathrm{~J} /(\mathrm{kg} \mathrm{K})$, these would yield a value for $\mu=1.67 \cdot 10^{-5} \mathrm{~m}^{2} / \mathrm{s}$.

Once the sizing matrix $\mathbf{K}^{\mathbf{S}}$ has been calculated, the dimensionless matrix $\widetilde{\mathbf{K}}$ is obtained by dividing element-wise the matrix $\mathbf{K}$ by $\mathbf{K}^{\mathbf{S}}$ (only applies for non-zero elements)

$\widetilde{K}_{i j}=\frac{K_{i j}}{K_{i j}^{S}}, \quad i, j: K_{i j} \neq 0, \quad i \neq j$.

$\widetilde{\mathbf{K}}$ represents the comparison between the conductive couplings of the detailed model and their estimated values obtained from the available data, such as geometry and thermal capacities.

\subsubsection{Boundary nodes isolation}

The boundary nodes have to be retained in the reduced model, and should not be condensed. They should remain as independent nodes, that is, "isolated". Therefore, they have to be artificially decoupled from the rest of the nodes in the model in order to process the adjacency matrix A adequately. To do so, the boundary nodes in the sizing matrix $\widetilde{\mathbf{K}}$ need to be marked. Basically, this means that all the related couplings to the boundary nodes will be artificially set to zero, so that they are not able to be condensed with any other node (the boundary nodes appear as if they were isolated). It is important to underline that this is done only in the $\widetilde{\mathbf{K}}$ matrix (the resulting matrix is called $\widetilde{\mathbf{K}}^{\mathbf{B}}$ ). It only affects the reduction scheme, but all the information regarding the conductive couplings between the boundary nodes and rest of the model is still available, and is consequently included in the reduced model.
In order to improve the processing of the conductive coupling matrix and the outcome of the model reduction, it is useful to isolate the nodes which are directly conductively coupled to the boundary nodes. These nodes are called "internal boundary nodes". Regardless of the aim of the model reduction (intensive internal calculations, delivery to an assembler of thermal models, etc.), the isolation of these internal nodes will generally allow for a better temperature correlation to be achieved in these nodes, which ultimately will yield a better heat fluxes correlation (because the conductive couplings between the boundary nodes and the internal boundary nodes are kept independent from the general reduction process). The process is applied in $\widetilde{\mathbf{K}}^{\mathbf{B}}$, and the resulting matrix is called $\widetilde{\mathbf{K}}^{\mathbf{I}}$.

Regarding the boundary nodes that are connected to the rest of the model only by means of radiative couplings, they are isolated as well, as their conductive couplings with the model are obviously zero.

\subsubsection{Filtering of the dimensionless conductive coupling matrix}

The matrix $\widetilde{\mathbf{K}}^{\mathrm{I}}$ is ready to be filtered by a value $p_{f}$, which represents a dimensionless threshold as follows

$\widetilde{K}_{i j}^{I, F}=\left\{\begin{array}{ll}1 & \widetilde{K}_{i j}^{I}>p_{f} \\ 0 & \widetilde{K}_{i j}^{I} \leqslant p_{f}\end{array}\right.$.

The resulting matrix $\widetilde{\mathbf{K}}^{\mathbf{1}, \mathbf{F}}$ is "binarized", a matrix with only ones and zeros, which contains the information of the nodes that are to be merged. The number of condensed nodes depends on $p_{f .} \widetilde{K}_{i j}^{I F}=1$ means a good connection, and therefore the connected nodes have to be included in the same condensed node. As the threshold value $p_{f}$ increases, more nodes appear as isolated in the model, and consequently the number of nodes in the reduced model is larger.

\subsection{Construction and process of temperature difference matrix}

The representativeness of the reduced model is evaluated by comparing the temperatures of the reduced model and of the detailed model. Therefore, if the nodes that form a condensed node are isothermal (or nearly isothermal), the reduced model will behave more similarly to the detailed one. Thus, it is reasonable to limit the temperature difference that two nodes can have in order for them to be included in the same condensed node.

\subsubsection{Building and filtering the temperature difference matrix}

The temperature difference matrix $\Theta$ is built with the nodal temperature vector $\mathbf{T}$ of the detailed model.

$\Theta_{i j}=\left|T_{i}-T_{j}\right|$.

The matrix $\Theta$ is ready to be filtered by a maximum temperature difference, $\Delta T_{\max }$. The resulting matrix is "binarized" in the same fashion as explained in Section 3.2.3, as follows,

$\Theta_{i j}^{F}= \begin{cases}0 & \Theta_{i j}>\Delta T_{\max } \\ 1 & \Theta_{i j} \leqslant \Delta T_{\max }\end{cases}$

Obviously, the greater the maximum temperature difference $\Delta T_{\max }$, the lower the number of nodes in the reduced model.

\subsection{Model condensation}

\subsubsection{Definition of the adjacency matrix}

With $\widetilde{\mathbf{K}}^{\mathbf{I}, \mathbf{F}}$ and $\boldsymbol{\Theta}^{\mathbf{F}}$ already built, it is necessary to merge both matrices in the adjacency matrix $\mathbf{A}$. This is achieved by multiplying both matrices element-wise

$A_{i j}=\widetilde{K}_{i j}^{I F} \cdot \Theta_{i j}^{F}$ 
where basically we are restricting the filtered dimensionless matrix $\widetilde{\mathbf{K}}^{\mathbf{I}, \mathbf{F}}$ with $\boldsymbol{\Theta}^{\mathbf{F}}$. Because of the definition of $\widetilde{\mathbf{K}}^{\mathbf{I} \mathbf{F}}$ and $\boldsymbol{\Theta}^{\mathbf{F}}$ in Eqs. (14) and (16), the adjacency matrix A will have only zeros and ones. It represents the thermal network as a graph, the nodes being the graph vertices and the conductive couplings the edges. The thermal network is an undirected graph, and $\mathbf{A}$ is a sparse adjacency symmetrical matrix. As mentioned in Section 3.1, the programming has been carried out using sparse matrices in CSR format [26].

\subsubsection{Connected components algorithm}

The model is reduced by running the connected components algorithm [20-22], so that the nodes are grouped according to their connections (the algorithm finds the nodes which are connected to each other). As a result, the algorithm returns the number of condensed nodes $n_{c}$, and the connected component labels array $\mathbf{S}_{\mathbf{c n}}$, in which the nodes belonging to the same connected component (thus, belonging to the same condensed node) have the same identifier.

\subsubsection{Sorting the matrix entities}

Sorting the original $\mathbf{K}$ and $\mathbf{R}$ matrices is not strictly necessary for the reduction process. However, arranging the thermal coupling matrices by condensed nodes order can give visual information about how the reduction process has grouped the nodes. To do this, a change of basis matrix $\mathbf{B}$ is necessary, which is built with the label array $\mathbf{S}_{\mathbf{c n}}$. Then, one can get the sorted versions of $\mathbf{K}$ and $\mathbf{R}$ as the following matrix products

$$
\begin{aligned}
& \mathbf{K}_{\text {sort }}=\mathbf{B} \cdot \mathbf{K} \cdot \mathbf{B}^{\mathbf{T}} \\
& \mathbf{R}_{\text {sort }}=\mathbf{B} \cdot \mathbf{R} \cdot \mathbf{B}^{\mathbf{T}}
\end{aligned}
$$

(See example in Section 4.1).

\subsubsection{Compacting the model}

To reduce the dimension of the input matrices and vectors a change of basis matrix, which is called restriction matrix $\mathbf{P}$, is needed. The dimensions of $\mathbf{P}$ are $n_{d}$ rows $\times n_{c}$ columns. The node order of $\mathbf{P}$ being the same as in $\mathbf{K}$, each row corresponds to a node, which will have a " 1 " in the column corresponding to the associated condensed node, and the rest of the elements in the row will be " 0 ". With the restriction matrix, and the original inputs, the matrix entities can be built for the reduced model

$$
\begin{aligned}
& \mathbf{K}^{\mathbf{R}}=\mathbf{P}^{\mathbf{T}} \cdot \mathbf{K} \cdot \mathbf{P} \\
& \mathbf{R}^{\mathbf{R}}=\mathbf{P}^{\mathbf{T}} \cdot \mathbf{R} \cdot \mathbf{P}^{\prime}
\end{aligned}
$$

Thus, for each condensed node, all of its conductive couplings with a neighbor are summed up. Same methodology is used for the radiative couplings. Actually, these combinations are the source of the deviation between the condensed nodes predicted temperatures (derived from the detailed thermal model) and those calculated by solving the reduced thermal model. The errors will be small as far as the condensed nodes are isothermal. Thus, the threshold values ( $p_{f}$ and $\Delta T_{\max }$ ) determine the error obtained in the reduction, considering the rest of the elements constant.

Also for the loads and thermal capacities vectors

$$
\begin{aligned}
& \mathbf{Q}^{\mathbf{R}}=\mathbf{P}^{\mathbf{T}} \cdot \mathbf{Q} \\
& \mathbf{C}^{\mathbf{R}}=\mathbf{P}^{\mathbf{T}} \cdot \mathbf{C}
\end{aligned}
$$

This operation assigns to each condensed node the sum of the thermal capacities of the nodes that belong to that condensed node. The same occurs for the thermal loads. The reduced system can be solved with the reduced matrices and vectors to obtain $\widehat{\mathbf{T}}^{\mathbf{R}}$. However, the correctness or accuracy of the reduction process needs to be evaluated. Thus, this matrix needs to be compared with the target temperatures $\overline{\mathbf{T}}^{\mathbf{D}}$, derived directly from the detailed model. One widely accepted standard in the industry is to calculate these temperatures as the thermal capacity weighted mean temperature values (it is also possible to weight the temperatures with nodal areas)

$\bar{T}_{i}^{D}=\frac{\sum_{j=1}^{m} T_{j} C_{j}}{\sum_{j=1}^{m} C_{j}}, \quad i=1, \ldots, n_{c}$,

where $m$ is the number of nodes that belong to condensed node $i$. Once the reduced model is solved, the temperatures derived from the detailed model, $\overline{\mathbf{T}}^{\mathbf{D}}$, are compared with those calculated with the reduced model, $\hat{\mathbf{T}}^{\mathbf{R}}$. The boundary nodes are directly assigned a temperature in the reduced model, as they were isolated in the adjacency matrix (Section 3.2.2).

Regarding the heat fluxes, the calculated values for the reduced model, $\widehat{\mathbf{H}}^{\mathbf{R}}$, are compared with the target values, those corresponding to the detailed model, $\mathbf{H}_{\mathrm{cb}}^{\mathbf{D}}$ and $\mathbf{H}_{\mathrm{rb}}^{\mathrm{D}}$. $\mathbf{H}_{\mathrm{cb}}^{\mathrm{D}}$ accounts for the conductive heat fluxes to boundary nodes, whereas the vector $\mathbf{H}_{\mathbf{r b}}^{\mathbf{D}}$ does so for the radiative heat fluxes.

\subsection{Validation of the reduced thermal model}

With the reduced matrices and vectors, the reduced system can be solved. $\overline{\mathbf{T}}^{\mathbf{D}}$ is used to set up the boundary conditions, and also as an initial condition if an iterative solver is employed. The thermal system is then solved in steady state conditions, one energy balance equation per node

$\sum_{j=1}^{n_{c}}\left[K_{i j}^{R}\left(\widehat{T}_{i}^{R}-\widehat{T}_{j}^{R}\right)+R_{i j}^{R}\left(\widehat{T}_{i}^{R^{4}}-\widehat{T}_{j}^{R^{4}}\right)\right]+Q_{i}^{R}=0, \quad i=1, \ldots, n_{c}, \quad i \neq j$.

The model is solved with a steady state solver. For the linearization of the radiative terms, the following algebraic identity is used (see [27])

$\left(\widehat{T}_{i}^{4}-\widehat{T}_{j}^{4}\right)=\left(\widehat{T}_{i}^{2}+\widehat{T}_{j}^{2}\right)\left(\widehat{T}_{i}+\widehat{T}_{j}\right)\left(\widehat{T}_{i}-\widehat{T}_{j}\right)$

Using Eq. (23) in the non-linear part of Eq. (22), one gets

$R_{i j}^{R}\left(\widehat{T}_{i}^{4}-\widehat{T}_{j}^{4}\right)=R_{i j}^{R}\left(\widehat{T}_{i}^{2}+\widehat{T}_{j}^{2}\right)\left(\widehat{T}_{i}+\widehat{T}_{j}\right)\left(\widehat{T}_{i}-\widehat{T}_{j}\right)=K_{i j}^{R^{+}}\left(\widehat{T}_{i}-\widehat{T}_{j}\right)$,

where

$K_{i j}^{R^{*}}=R_{i j}^{R}\left(\widehat{T}_{i}^{2}+\widehat{T}_{j}^{2}\right)\left(\widehat{T}_{i}+\widehat{T}_{j}\right)$.

The values for $\mathbf{K}^{\mathbf{R}^{*}}$ are obtained with the temperatures calculated for the current step. Then, the system is solved, and the latest temperatures are compared with those corresponding to the previous step. Once the model has been solved, the results must be compared with those coming from the detailed model. For the temperatures, the absolute difference will have to be less than a predefined value, $\delta_{\max }$. Hence, with the reduced model derived temperatures $\overline{\mathbf{T}}^{\mathbf{D}}$, and the reduced model calculated temperatures $\widehat{\mathbf{T}}^{\mathbf{R}}$, it can be checked that the difference is below the maximum allowable value $\delta_{\max }$.

$\delta_{i}=\left|\bar{T}_{i}^{D}-\widehat{T}_{i}^{R}\right|<\delta_{\max }, \quad i=1, \ldots, n_{c}$.

Regarding the heat fluxes exchanged with the boundary nodes, the heat fluxes vectors will have as many components as the number of boundary nodes, $n_{b}$. The difference can be expressed in terms of percentage or in absolute numbers, depending on the heat flux value (the limit is defined by $q_{t i m}$ ). For the conductive heat fluxes 


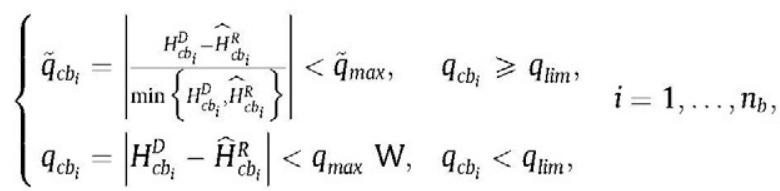

and for the radiative heat fluxes

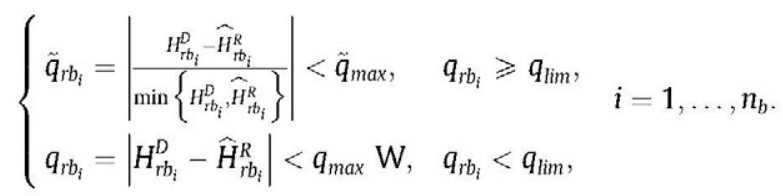

\section{Test model}

In order to analyze the application of the method to a real problem with a large number of nodes, let us consider the thermal model of the Focal Plane Assembly (FPA) of the Polarimetric and Helioseismic Imager ( $\mathrm{PHI}$ ) instrument. $\mathrm{PHI}$ will be on board the ESA mission Solar Orbiter, to be launched in 2018 [28]. The FPA detailed thermal model consists of 1072 nodes and 457 geometric primitives, and has been entirely set up with ESATAN-TMS [10]. The FPA is a camera system, with a detector, front-end electronics, and a cold finger which connects the camera to the PHI Optics Unit cold element by means of a thermal strap. It also has an aluminum

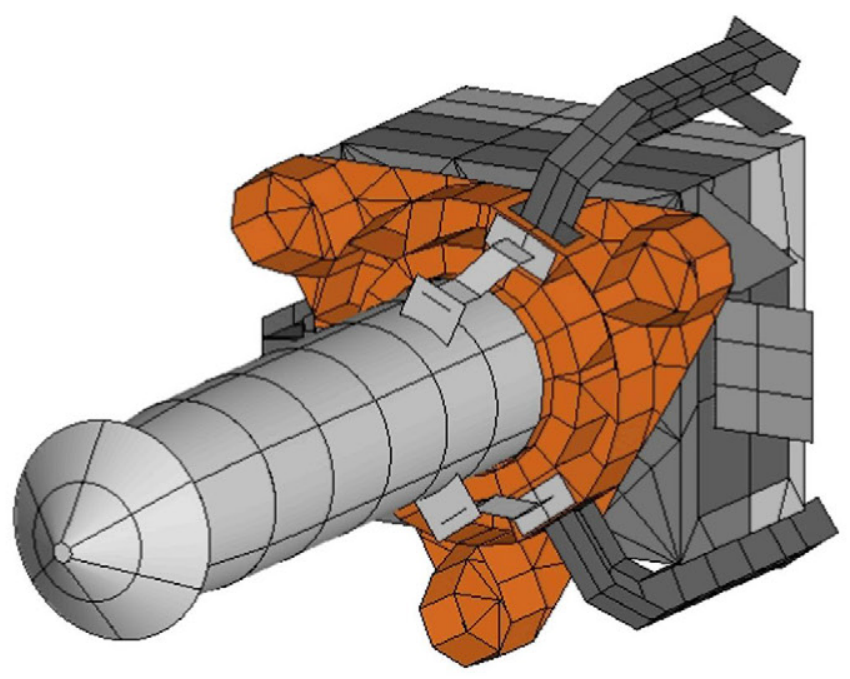

Fig. 3. PHI Optics Unit FPA GMM

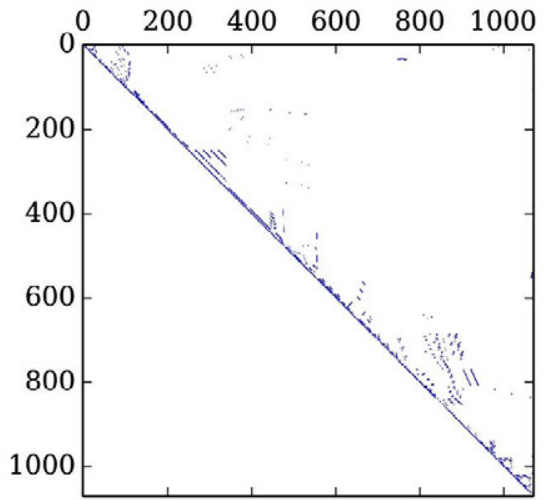

(a) housing, and an aluminum tube with some lenses. The PHI FPA geometrical mathematical model (GMM) is shown in Fig. 3.

\subsection{Reduction of the thermal mathematical model}

The conductive and radiative coupling matrices are shown in Fig. 4 as imported from the ESATAN-TMS TMD (Thermal Model Data) file. Matrix $\mathbf{K}$ has 2367 conductive couplings, whereas $\mathbf{R}$ has 299,252 . The matrix $\mathbf{R}$ is very dense not only because it represents the radiative couplings, but also due to the accuracy parameters used in the radiative calculations. The matrices are symmetric, so the program only works with upper triangular matrices. All the graphics related to the reduction process have been done with Matplotlib [29].

The model has four boundary nodes. If they are isolated, and the strongly connected components algorithm on the resulting matrix $\mathbf{K}^{\mathbf{B}}$ is run, the minimum number of condensed nodes is found to be 6 (including the 4 boundary nodes and 2 nodes which are decoupled from each other). The maximum ratio $r_{r}$, which can be got according to Eq. (2), is 0.998 (from 1072 to 6). This is a theoretical limit, but in order to correlate the models, this value of $r_{r}$ will have to be lower.

To prepare the matrix $\mathbf{K}$ for the filtering, $\mu$ has been given the value calculated in Section $3.2 .1\left(\mu=1.67 \cdot 10^{-5} \mathrm{~m}^{2} / \mathrm{s}\right)$. Then, with $\mu$, together with the distance matrix $\mathbf{D}$ and the capacitance matrix $\mathbf{G}$, the sizing matrix $\mathbf{K}^{\mathbf{S}}$ can be obtained. The distribution of the conductive couplings matrices $\mathbf{K}$ and $\mathbf{K}^{\mathbf{S}}$ is shown in Fig. 5.

In Fig. 5, it can be seen that most of the couplings for matrix $\mathbf{K}$ are concentrated in the range $10^{-1}-10^{0} \mathrm{~W} / \mathrm{K}$, whereas for matrix $\mathbf{K}^{\mathbf{S}}$ they are mostly concentrated in the range $10^{1}-10^{2} \mathrm{~W} / \mathrm{K}$. This means that, according to the value chosen for parameter $\mu$ and the magnitudes used to estimate $\mathbf{K}^{\mathbf{S}}$, the model has a "poor" conductive behavior ( $\lambda$ has a generic value of $50 \mathrm{~W} /(\mathrm{m} \mathrm{K})$ ). This appraisal would change in the moment $\mu$ is changed, but still it gives an idea of how conductive the model is if it is compared with the estimator.

Once the dimensionless coupling $\widetilde{\mathbf{K}}^{\mathbf{I}}$ and temperature $\Theta$ matrices have been created, it is possible to set up the adjacency matrix A, filter the system and compact the model. Before that, it was decided to do a preliminary study regarding how the reduction ratio $r_{r}$ varies with the different filtering levels. Fig. 6 shows the variation of $r_{r}$ with $p_{f}$ and $\Delta T_{\max }$ independently. This figure confirms what was explained in Sections 3.2.3 and 3.3.1 relative to the behavior of $r_{r}$ as a function of $p_{f}$ and $\Delta T_{\max }$.

As the reduction ratio $r_{r}$ is a function of both $p_{f}$ and $\Delta T_{\max }$, to analyze the influence of these two parameters an appropriate method is to create a contour plot of the function $r_{r}=f\left(p_{f}, \Delta T_{\max }\right)$,

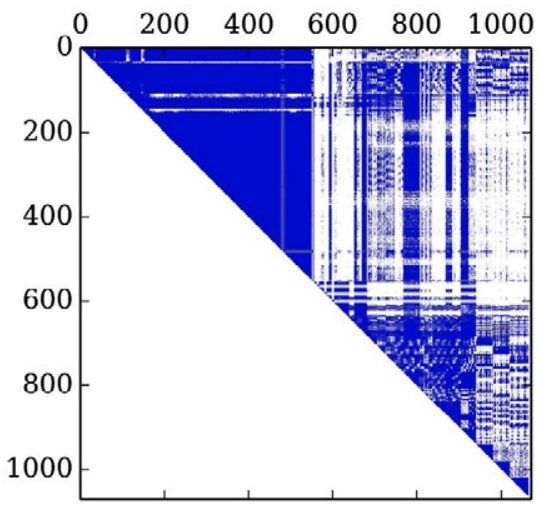

(b)

Fig. 4. Sparse graphical representation of (a) matrix $\mathbf{K}$ and (b) matrix $\mathbf{R}$. 


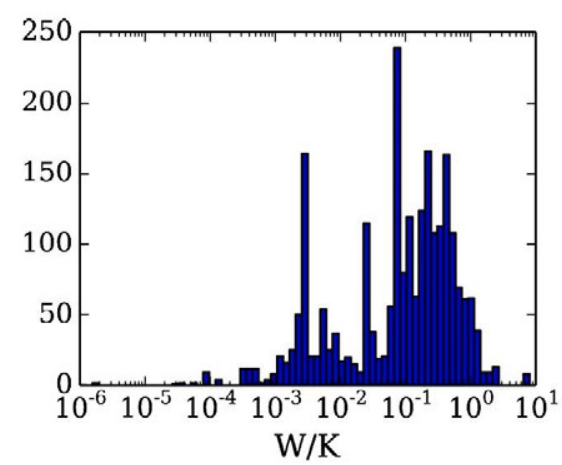

(a)

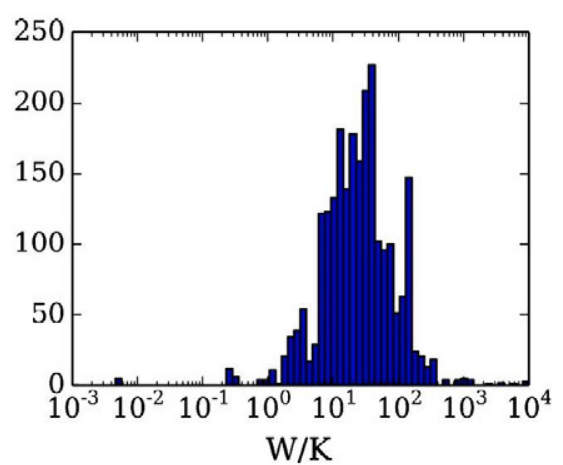

(b)

Fig. 5. Conductive couplings histograms of (a) matrix $\mathbf{K}$ values and (b) matrix $\mathbf{K}^{\mathbf{S}}$ values.

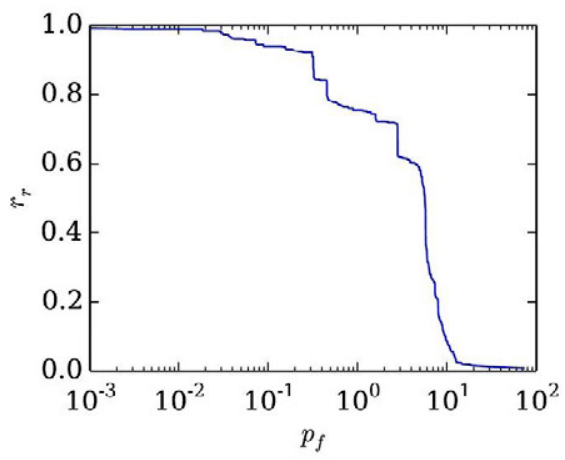

(a)

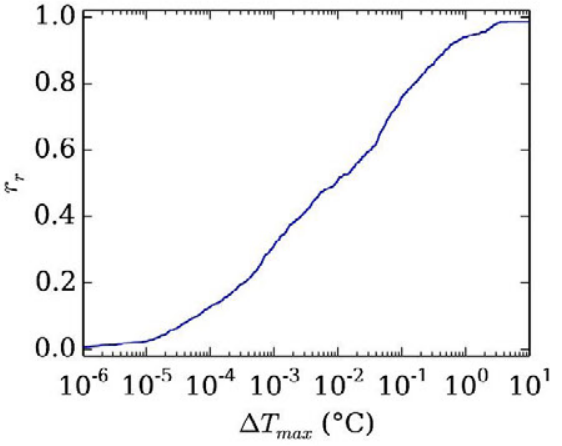

(b)

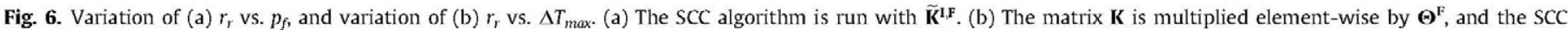
algorithm is run with the resulting matrix.

as shown in Fig. 7. This way, a combination of $p_{f}$ and $\Delta T_{\max }$ suitable to get the desired value of $r_{r}$ can be selected. Furthermore, it is necessary to determine if the model correlation is good enough. To do this, a correlation criteria for the reduced model should be defined.

For the sake of simplicity, only one is used for all the parts: $\delta_{\max }=3 \mathrm{~K}$. Regarding the heat flux exchanged with the boundary nodes, the chosen criteria are:

a. For absolute heat flow less than $q_{\text {lim }}=1 \mathrm{~W}$, difference must be less than $0.1 \mathrm{~W}$.

b. For absolute heat flow more than $q_{\text {lim }}=1 \mathrm{~W}$, difference must be less than $10 \%$.

Therefore, the value for $q_{\max }$ is 0.1 and $\tilde{q}_{\max }$ is $10 \%$. Fig. 7 shows the function $r_{r}=f\left(p_{f}, \Delta T_{\max }\right)$, together with the correlation status for each pair of values $p_{f}$ and $\Delta T_{\max }$. The successful correlation implies that the three conditions (temperature difference, conductive and radiative heat fluxes differences) are satisfied. The values for $p_{f}$ and $\Delta T_{\max }$ cover almost the full range of both magnitudes, as shown in Fig. 6. The most extreme values have been omitted. As can be seen in Fig. 6 , the extreme values of the variation range for $p_{f}$ and $\Delta T_{\max }$ produce almost no change in the reduction ratio $r_{r}$.

Hence, it can be seen from the results shown in Fig. 7 that it is not possible to have a reduction ratio $r_{r}$ of more than $0.75-0.80$ that yields a successful correlation (with the current correlation criteria). This is due to the PHI FPA model design idiosyncrasy. Other models could yield greater values of $r_{r}$ that satisfy the correlation criteria. In order to study in detail this model reduction in particular, let us plot the same Fig. 7 but zooming in on the range of $r_{r}=[0.50,0.90]$. This zooming in, together with the display of temperature and heat flux difference maps, is shown in Fig. 8.

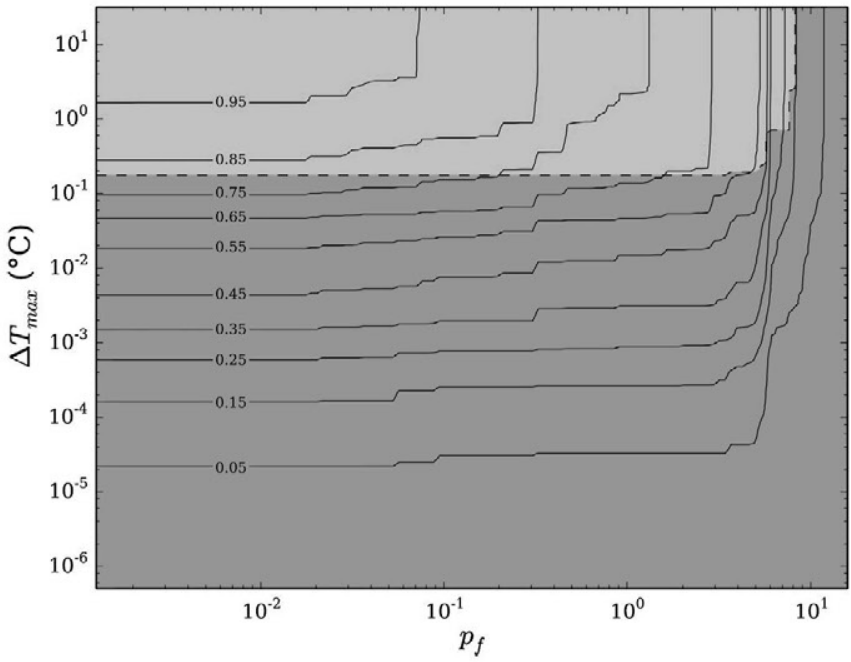

Fig. 7. Contour plot of the function $r_{r}=f\left(p_{f}, \Delta T_{\max }\right)$. Black solid lines represent constant $r_{r}$, whose value is indicated inline. Lighter gray zone indicates successful correlation for the resulting reduced model. Darker gray colored zone indicates failed correlation for the resulting reduced model. Dashed black line indicates the separation between both zones, being effectively the limit for a successful correlation.

As shown in Fig. 6, the variation of $r_{r}$ with $p_{f}$ is steep, whereas the variation of $r_{r}$ with $\Delta T_{\max }$ is more gradual. This behavior can be observed in Fig. 7. For the same value of $\Delta T_{\max }$ and $r_{r}$, it is better to choose a higher value of $p_{f}$, as the potential number of conductive couplings will be lower. We can also see that the most restric- 


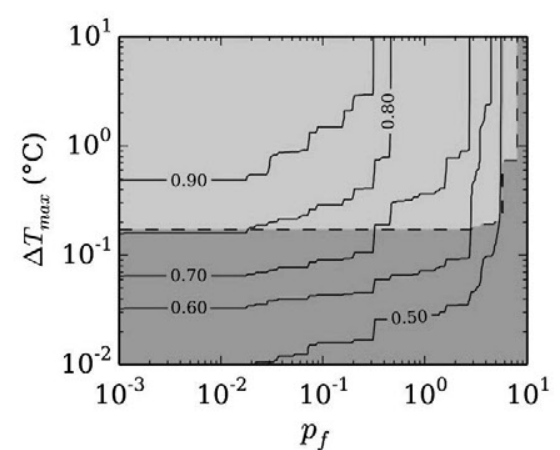

(a)

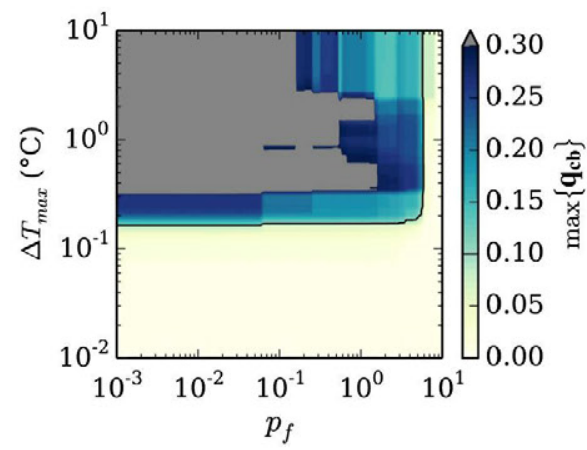

(c)

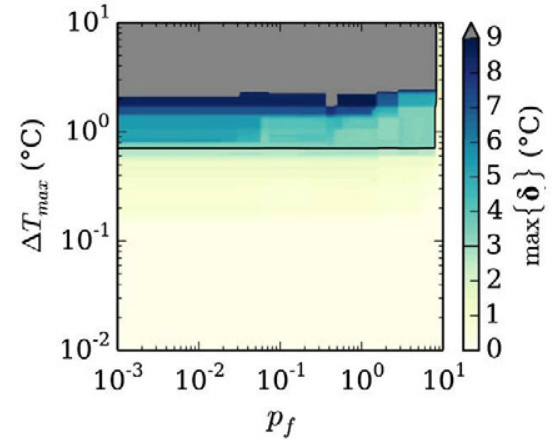

(b)

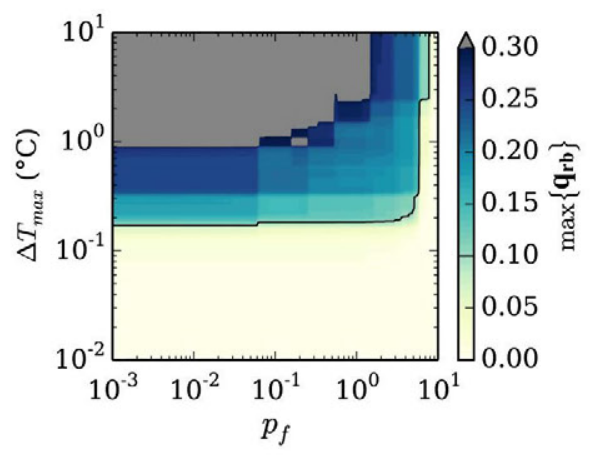

(d)

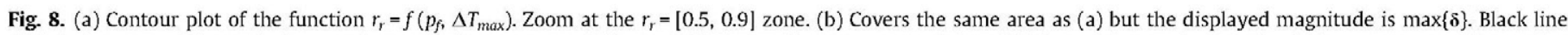

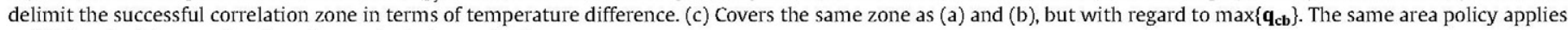
to (d), but in this case the plotted magnitude is $\max \left\{\mathbf{q}_{\mathbf{r b}}\right\}$.

tive criteria for the correlation are the heat flux differences (except for a small area where $p_{f} \approx 10^{1}$ and $\Delta T_{\max }$ ranges between $10^{\circ}$ and $\left.10^{1}{ }^{\circ} \mathrm{C}\right)$.

Let us select a combination of values for $p_{f}$ and $\Delta T_{\max }: p_{f}=0.1$ and $\Delta T_{\max }=0.15^{\circ} \mathrm{C}$. After applying the SCC algorithm, these values yield a $n_{c}=272$ nodes. With $n_{b}=4$, Eq. (2) gives $r_{r}=0.75$.

The outcome of the SCC algorithm allows the restriction matrix $\mathbf{P}$ to be built (Fig. 9), as well as the sorted matrices $\mathbf{K}_{\text {sort }}$ and $\mathbf{R}_{\text {sort }}$ (Fig. 10). With the restriction matrix $\mathbf{P}$ the reduced matrices $\mathbf{K}^{\mathbf{R}}$ and $\mathbf{R}^{\mathbf{R}}$ can be built (Fig. 11).

With the values of $p_{f}$ and $\Delta T_{\max }$ chosen, it is already known by looking at Fig. 8, that the correlation of the reduced model with the detailed model will be within the limits. The reduced system to find out the exact values for the temperature and heat fluxes differences can now be solved. Fig. 12 shows the components for the temperature difference vector $\delta$. As can be deduced from this figure, the maximum value is around $0.35 \mathrm{~K}$, and the minimum is around $-0.25 \mathrm{~K}$.

Regarding the conductive heat flux differences, the values from the detailed model $\mathbf{H}_{\mathbf{c b}}^{\mathbf{D}}$ and those calculated with the reduced model $\widehat{\mathbf{H}}_{\mathrm{cb}}^{\mathbf{R}}$ are

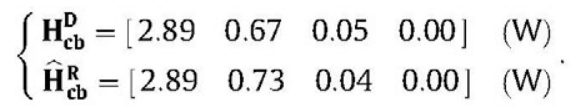

If both vectors are compared, it can be seen that the maximum difference occurs for the second boundary node. According to the definition given in Eq. (27), together with our criteria about heat flux differences, it can be seen that the goal value is smaller than $1 \mathrm{~W}$, and consequently the difference must be given in absolute values (and not in percentage),

$q_{c b_{2}}=\left|H_{c b_{2}}^{D}-\widehat{H}_{c b_{2}}^{R}\right|=0.06 \mathrm{~W}<q_{\max }=0.1 \mathrm{~W}$,

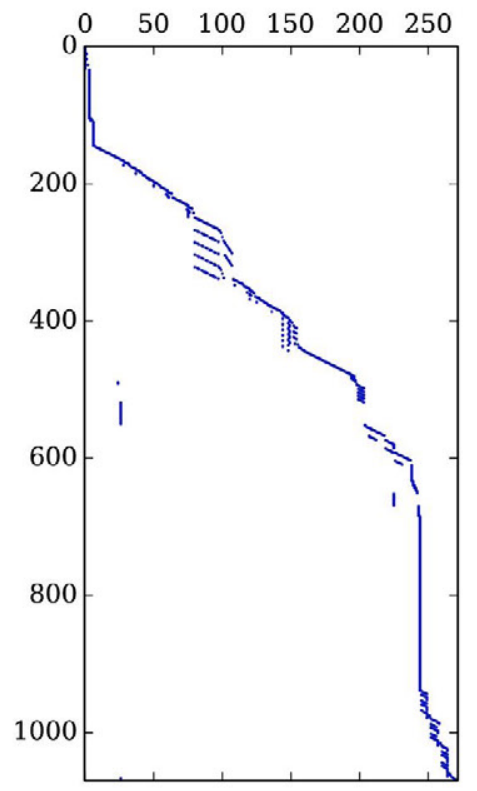

Fig. 9. Sparse graphical representation of restriction matrix $\mathbf{P}$.

and for radiative heat fluxes

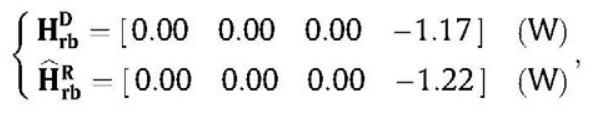

where the difference, according to Eq. (28) is

$$
\tilde{q}_{r b_{4}}=\left|\frac{H_{r b_{4}}^{D}-\widehat{H}_{r b_{4}}^{R}}{\min \left\{H_{r b_{4}}^{D}, \widehat{H}_{r b_{4}}^{R}\right\}}\right|=4.3 \%<\tilde{q}_{\max }=10 \% \text {. }
$$




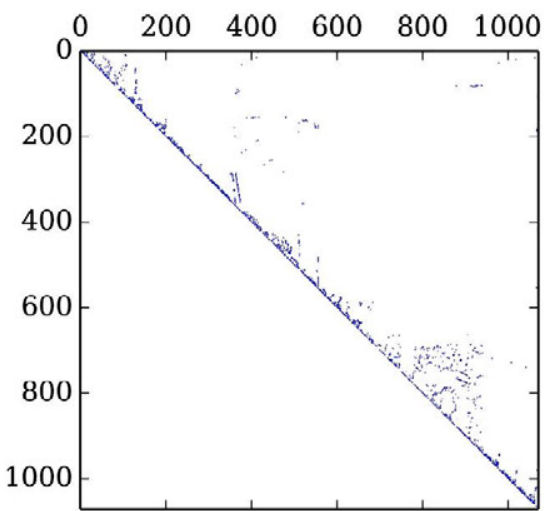

(a)

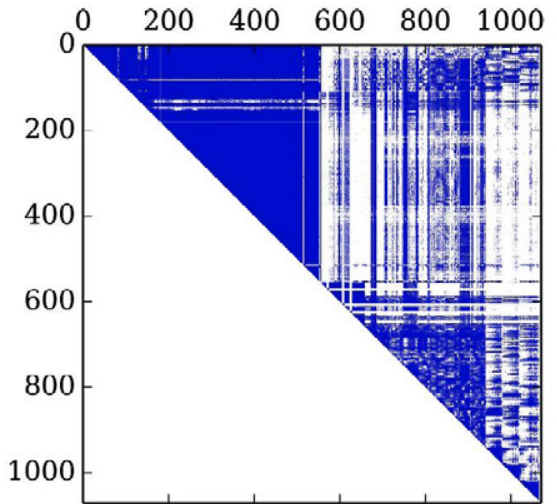

(b)

Fig. 10. Sparse graphical representation of (a) matrix $\mathbf{K}_{\text {sort }}$ and (b) matrix $\mathbf{R}_{\text {sort }}$. The matrices are symmetric, so the program only works with upper triangular matrices.

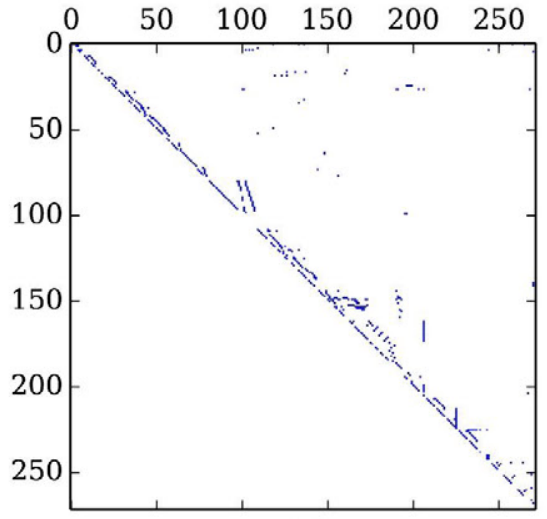

(a)

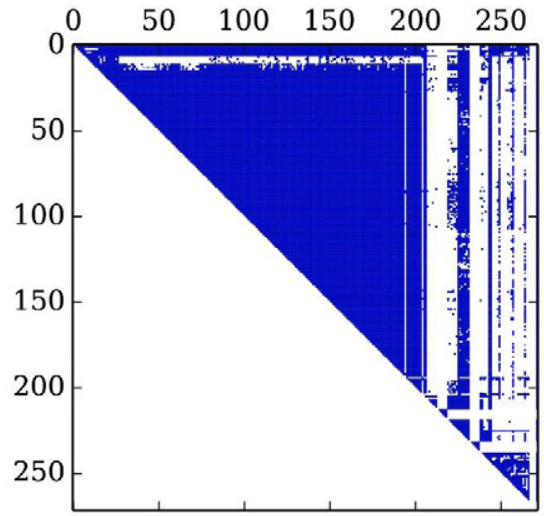

(b)

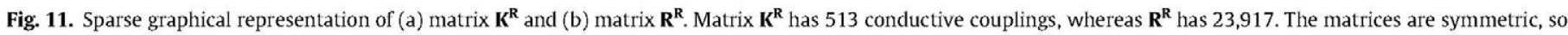
the program only works with upper triangular matrices.

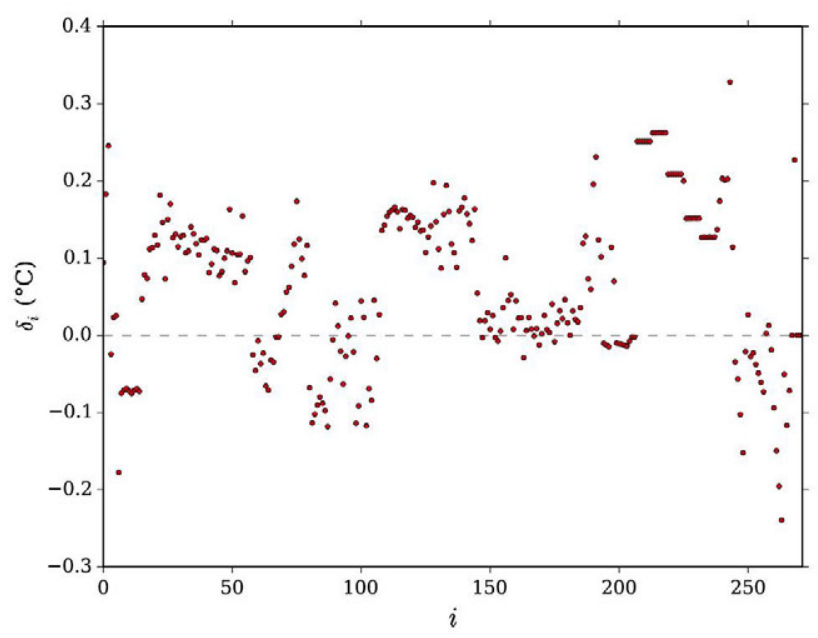

Fig. 12. Components of vector $\boldsymbol{\delta}$.

The program takes $180 \mathrm{~min}$ in a mid-2014 desktop computer to run 25,350 cases (reduced models obtained with different combinations of $p_{f}$ and $\Delta T_{\max }$ ), in order to plot Figs. 7 and 8 .

\subsection{Reduction of the geometrical mathematical model}

Based on the reduction scheme developed in Section 4.1, it is necessary to reduce the GMM accordingly. Although it is possible to develop a kind of automatic algorithm to reduce automatically the GMM [17], we believe that the manual reduction serves its purpose, and it is not as time consuming as the TMM reduction. With the adequate tools to get the information from the TMM reduction scheme, the GMM reduction can be very effective.

\section{Conclusions}

The method presented in this paper works well with a broad selection of thermal models. It is focused on internally mounted space scientific instrument thermal models, although formally the method could be used in anyone. The method uses the steady-state conditions for the reduction process, finding the condensed nodes based on thermal conductance values and temperature similarities. It is fast, intuitive and preserves all the physical characteristics of the original thermal model. The value of the maximum reduction ratio $r_{r}$ for which the correlation is successful depends on the model, but, according to the tests carried out, it seems quite difficult to achieve reductions with a reduction ratio of $90 \%$ or more. If it is necessary to reach higher levels of reduction, then one option could be to use optimization and stochastic methods on top of the reduction method explained in this paper [30-32].

The transient correlation is beyond the scope of this study, but first studies show that the reduced models obtained by this technique behave reasonably well. Other aspects which have not been dealt with here are multi-layer insulation (MLI), thermo-electric 
coolers and other special thermal control technologies models reduction.

\section{Acknowledgements}

This work has been supported by the Spanish Ministerio de Economía y Competitividad, Projects AYA2012-39636-C06-04 and ESP2013-47349-C6-4-R. The first author was awarded a mobility grant from Consejo Social of Universidad Politécnica de Madrid, which supported his research stay carried out at the Max-PlanckInstitut für Sonnensystemforschung, Göttingen, Germany, from April to June 2014. 